\title{
Le Centre canadien de ressources et d'échange sur les données probantes en vaccination (CANVax) : Une nouvelle ressource permettant de résumer les données probantes sur la vaccination
}

\author{
Noni E MacDonald ${ }^{1 *}$, Eve Dubé2
}

\section{Résumé}

Les progrès scientifiques réalisés autour de la mise au point, de l'utilisation et de l'amélioration des pratiques visant à communiquer les avantages des vaccins sont rapides, et se tenir au courant du vaste ensemble de données à cet égard représente un défi. L'augmentation du nombre de vaccins et le déclin des maladies évitables par la vaccination ont déplacé l'attention du public sur les risques de la vaccination plutôt que sur les risques des maladies. Au Canada et ailleurs, un nombre croissant de parents choisissent de retarder ou de refuser certains vaccins, voire tous, pour leurs enfants, ce qui entraîne un déclin de la protection de la collectivité contre les maladies évitables par vaccination et une augmentation du nombre d'éclosions de maladies évitables par la vaccination. Les données probantes indiquent que la notion d'hésitation à la vaccination contribue à une meilleure compréhension des décisions en matière de vaccination, car elle dépasse les attitudes binaires pour ou contre la vaccination et reconnaît un spectre de croyances et de comportements connexes qui occupe l'espace entre les deux pôles. À une époque où croît la résistance antimicrobienne face aux infections, la protection qu'apporte la vaccination est plus importante que jamais.

Le Centre canadien de ressources et d'échange sur les données probantes en vaccination (CANVax) est une base de données de ressources en ligne qui soutient les activités de vaccination et de promotion visant à améliorer l'acceptation et l'adoption de la vaccination au Canada. II s'occupe à la fois de l'identification des ressources exactes et fiables et de la création de nouvelles ressources par un groupe multidisciplinaire de professionnels.

Ce numéro du Relevé des maladies transmissibles au Canada (RMTC) contient le premier d'une série d'articles intitulés "Brèves du CANVax » préparés par des experts ayant effectué des études de portée et des analyses environnementales, et évalué les données probantes les plus rigoureuses. Les Brèves du CANVax visent à attirer l'attention sur les questions actuelles et émergentes en offrant de courts résumés des meilleures et plus récentes données probantes disponibles pour aider les professionnels des soins de santé et de la santé publique de première ligne à optimiser le taux de vaccination au Canada. Les Brèves du CANVax seront publiées dans le RMTC pendant toute l'année 2020.
Cette oeuvre est mise à la disposition selon les termes de la licence internationale Creative Commons Attribution 4.0

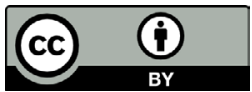

Affiliations

1 Département de pédiatrie, Université Dalhousie, Centre de soins de santé IWK, Halifax, NS

${ }^{2}$ Institut national de la santé publique du Québec,

Québec, QC

${ }^{\star}$ Correspondance :

noni.macdonald@dal.ca
Citation proposée : MacDonald NE, Dubé E. Nouvelle ressource permettant de résumer les données sur la vaccination provenant du Centre canadien de ressources et d'échange sur les données probantes en vaccination (CANVax). Relevé des maladies transmissibles au Canada 2020;46(1):17-21.

https://doi.org/10.14745/ccdr.v46i01a03f

Mots-clés : vaccins, réticence à la vaccination, politique fondée sur des données probantes, éclosions de maladies évitables par la vaccination, CANVax 


\section{Introduction}

Les progrès scientifiques réalisés autour de la mise au point $t$ de I'utilisation de vaccins ont été rapides au cours des 40 dernières années (1). Au Canada, le nombre de vaccins inclus dans le programme public de vaccination pour les enfants de 18 ans et moins a plus que doublé entre 1980 et 2019, de même que le vaccin a passé de huit à 17 antigènes (2). Non seulement le nombre de vaccins est-il maintenant plus élevé, mais le nombre de vaccins donnés en une même visite a lui aussi augmenté. Ainsi, jusqu'à quatre vaccins différents peuvent être donnés dans une seule visite de vaccination des enfants selon le programme de vaccination provincial et territorial (3).

Les calendriers de vaccination deviennent de plus en plus complexes, tout comme les besoins et les attentes des patients et des professionnels de la santé en ce qui concerne l'innocuité et l'efficacité des vaccins. Pour certaines personnes, I'augmentation du nombre de vaccins et le déclin des maladies évitables par la vaccination ont déplacé l'attention sur les risques de la vaccination plutôt que sur les risques des maladies (4). Au Canada et ailleurs, un nombre croissant de parents choisissent de différer ou de refuser certains vaccins, voire tous, pour leurs enfants, ce qui entraîne un déclin de la protection de la collectivité contre les maladies évitables par vaccination et d'importantes éclosions de maladies évitables par la vaccination (5-9). Malheureusement, les taux déclarés d'adoption au Canada sont inférieurs aux objectifs nationaux et internationaux (10).

Au Canada, entre $15 \%$ et $20 \%$ des parents environ sont inquiets à l'idée d'accepter des vaccins pour leurs enfants, certains décidant de retarder, voire de refuser les vaccins, en tout ou en partie $(5,11,12)$. Étant donné que les parents qui affichent une hésitation à la vaccination ne sont pas uniformément répartis dans la population, mais sont souvent regroupés géographiquement en raison des valeurs et des modes de vie qu'ils partagent, nous sommes maintenant en présence de terreaux fertiles pour d'importantes éclosions de maladies évitables par la vaccination (7-9).

La récente augmentation des éclosions de maladies évitables par la vaccination a eu lieu en même temps que la montée de la résistance antimicrobienne. La résistance antimicrobienne menace l'efficacité de la prévention et du traitement d'un nombre d'infections qui ne cesse d'augmenter (13). En 2019, l'Organisation mondiale de la santé a identifié la résistance antimicrobienne et la réticence à la vaccination comme faisant partie des 10 principales menaces à la santé mondiale (14), ce qui rend l'optimisation des taux de vaccination plus importante que jamais.

Dans le présent éditorial, nous présentons le phénomène de I'hésitation à la vaccination et ses déterminants; nous décrivons le Centre canadien de ressources et d'échange sur les données probantes en vaccination (CANVax), qui constitue une nouvelle ressource offrant des informations organisées sur les vaccins destinées aux professionnels des soins de santé et de la santé publique de première ligne ainsi que d'autres services éducatifs; et nous présentons une série de Brèves du CANVax qui seront publiées dans le Relevé des maladies transmissibles au Canada (RMTC) tout au long de 2020, en commençant par le présent numéro.

\section{Hésitation à la vaccination}

L'hésitation à la vaccination, qu'on peut définir comme « le délai d'acceptation ou le refus du vaccin malgré la disponibilité de services de vaccination » (12), fait l'objet d'une attention croissante au niveau international. La portée de l'hésitation à la vaccination inclut les instances où " l'acceptation du vaccin dans un contexte en particulier est plus faible que prévu, étant donné la disponibilité des services de vaccination » (12).

La notion d'hésitation à la vaccination contribue à une meilleure compréhension des décisions en matière de vaccination, car elle dépasse les attitudes binaires pour ou contre la vaccination et reconnaît un spectre de croyances et de comportements connexes qui occupe l'espace entre les deux pôles. Une personne hésitante à la vaccination peut retarder, hésiter, mais quand même accepter, ou refuser, les vaccins, en tout ou en partie.

Les décisions en matière de vaccination sont complexes et multidimensionnelles, et peuvent être propres à un vaccin en particulier.

Au niveau individuel, les études ont mis l'accent sur les facteurs associés à l'acceptation ou au refus de la vaccination, et ont notamment identifié les déterminants suivants :

- crainte des effets secondaires

- conception de la santé et de la prévention des maladies

- préférence pour la santé «naturelle »

- mauvaise opinion de l'efficacité et de l'utilité des vaccins

- expériences passées négatives avec les services de vaccination

- manque de sensibilisation ou de connaissances à propos de la vaccination (4)

L'Organisation mondiale de la santé ramène à trois grandes catégories les différents facteurs qui mènent à la réticence à la vaccination (12) :

- Complaisance : les risques perçus des maladies évitables par la vaccination sont faibles et la vaccination n'est pas jugée nécessaire

- Commodité : qualité réelle et/ou perçue du service et degré auquel les services de vaccination sont offerts à un moment, dans un lieu et dans le contexte culturel qui sont pratiques et confortables 
- Confiance : confiance dans l'efficacité et l'innocuité des vaccins; dans le système qui les offre, notamment dans la fiabilité et la compétence des services de santé et des professionnels de la santé; et dans les motivations des responsables des politiques qui déterminent quels vaccins sont nécessaires, quand et où

L'intérêt grandissant pour l'hésitation à la vaccination, et plus généralement pour l'acceptation de la vaccination, génère un nombre de publications qui ne cesse d'augmenter. Se tenir au courant des importantes études nationales et internationales et du vaste ensemble d'articles de recherche révisés par les pairs et portant sur l'acceptation et l'adoption de la vaccination représente un défi.

\section{CANVax}

Le CANVax est une nouvelle base de données organisées de ressources en ligne qui soutient les activités de promotion et de planification des programmes de vaccination visant à améliorer I'acceptation et l'adoption de la vaccination au Canada (15). Le CANVax a été mis au point par l'Association canadienne de santé publique (ACSP) avec le financement de l'Agence de la santé publique du Canada. II offre des ressources organisées provenant du Canada et du monde entier, des fonctions interactives, des mises à jour de formation et de nouvelles ressources. Le CANVax est maintenant membre du Réseau pour la sécurité des vaccins, de l'Organisation mondiale de la santé; il s'agit d'un réseau international de sites Web approuvés qui fournissent des informations fiables sur la sécurité des vaccins (16).

\section{Ressources organisées}

Le CANVax héberge une collection de produits et de ressources sélectionnés fondés sur des données probantes permettant aux professionnels de la santé publique d'accéder plus facilement aux ressources qui éclaireront leur planification et leurs activités. II comprend une grande base de données portant sur cinq grands domaines : la prise de décisions en matière de vaccination; le contrôle et la surveillance; l'innocuité et la mise au point des vaccins; la planification et la prestation de programmes; et les politiques. Chaque domaine comprend plusieurs sujets, et pour chacun des sujets il y a des ressources sur les connaissances de base, des outils de mise en œuvre et des données probantes, ainsi que le nom des principaux influenceurs ou chefs de file du secteur (tableau 1).

Comment ça marche? Le CANVax est soutenu par un groupe multidisciplinaire d'experts possédant des compétences et des connaissances en santé publique, en maladies infectieuses, en anthropologie médicale, en pédiatrie, en médecine interne, en sociologie, en informatique, en médias sociaux et en bibliothéconomie. Ce groupe passe en revue les ressources, produits et outils canadiens et internationaux avant leur inclusion sur le site Web du CANVax.

\section{Tableau 1 : Résumé des domaines et sujets figurant dans la base de données du CANVax}

\begin{tabular}{|c|c|c|}
\hline Domaines & Sujets & Ressources \\
\hline \multirow{6}{*}{$\begin{array}{l}\text { Prise de } \\
\text { décisions en } \\
\text { matière de } \\
\text { vaccination }\end{array}$} & \multirow{3}{*}{$\begin{array}{l}\text { Hésitation à la } \\
\text { vaccination }\end{array}$} & $\begin{array}{l}\text { Compréhension de la réticence } \\
\text { et de la prise de décisions en } \\
\text { matière de vaccination }\end{array}$ \\
\hline & & Idées fausses \\
\hline & & $\begin{array}{l}\text { Médecine complémentaire et } \\
\text { alternative }\end{array}$ \\
\hline & \multirow{3}{*}{$\begin{array}{l}\text { Acceptation de la } \\
\text { vaccination }\end{array}$} & $\begin{array}{l}\text { Compréhension de } \\
\text { l'acceptation et de l'adoption } \\
\text { de la vaccination }\end{array}$ \\
\hline & & Consultation et communication \\
\hline & & $\begin{array}{l}\text { Gestion de l'angoisse et de la } \\
\text { douleur }\end{array}$ \\
\hline \multirow{6}{*}{$\begin{array}{l}\text { Veille } \\
\text { scientifique } \\
\text { et } \\
\text { surveillance }\end{array}$} & $\begin{array}{l}\text { Veille scientifique } \\
\text { et surveillance de la } \\
\text { vaccination }\end{array}$ & $\begin{array}{l}\text { Couverture et objectifs de la } \\
\text { vaccination }\end{array}$ \\
\hline & \multirow{2}{*}{$\begin{array}{l}\text { Éclosions et } \\
\text { pandémies }\end{array}$} & Éclosions \\
\hline & & Pandémies de grippe \\
\hline & \multirow{3}{*}{$\begin{array}{l}\text { Veille scientifique } \\
\text { des maladies } \\
\text { évitables par la } \\
\text { vaccination }\end{array}$} & $\begin{array}{l}\text { Veille scientifique provinciale et } \\
\text { territoriale }\end{array}$ \\
\hline & & Veille scientifique nationale \\
\hline & & Veille scientifique mondiale \\
\hline \multirow{4}{*}{$\begin{array}{l}\text { Innocuité et } \\
\text { mise au point } \\
\text { des vaccins }\end{array}$} & \multirow{2}{*}{$\begin{array}{l}\text { Innocuité des } \\
\text { vaccins }\end{array}$} & Innocuité des vaccins \\
\hline & & $\begin{array}{l}\text { Effets secondaires suivant la } \\
\text { vaccination (ESSV) }\end{array}$ \\
\hline & \multirow{2}{*}{$\begin{array}{l}\text { Recherche et } \\
\text { développement }\end{array}$} & Ingrédients des vaccins \\
\hline & & Mise au point des vaccins \\
\hline \multirow{7}{*}{$\begin{array}{l}\text { Planification } \\
\text { et prestation } \\
\text { des } \\
\text { programmes }\end{array}$} & \multirow{2}{*}{$\begin{array}{l}\text { Promotion et } \\
\text { communications }\end{array}$} & Marketing et campagnes \\
\hline & & Communications \\
\hline & \multirow{2}{*}{$\begin{array}{l}\text { Prestation et } \\
\text { évaluation des } \\
\text { programmes }\end{array}$} & Consentement éclairé \\
\hline & & Cliniques \\
\hline & Gestion des vaccins & Stockage et manipulation \\
\hline & $\begin{array}{l}\text { Gestion des } \\
\text { éclosions et des } \\
\text { crises }\end{array}$ & Communications en cas de crise \\
\hline & $\begin{array}{l}\text { Perfectionnement } \\
\text { professionnel }\end{array}$ & Éducation et formation \\
\hline Politique & $\begin{array}{l}\text { Politiques de } \\
\text { vaccination }\end{array}$ & $\begin{array}{l}\text { Activités et vaccination } \\
\text { obligatoires }\end{array}$ \\
\hline
\end{tabular}

\section{Fonctions interactives}

Tous les utilisateurs peuvent télécharger n'importe quelle ressource. En outre, les fonctions interactives suivantes sont offertes lorsqu'un utilisateur s'inscrit au CANVax :

- Partager - partagez facilement une ressource avec un collègue en utilisant les médias sociaux ou le courriel

- Commenter - laissez des commentaires sur les ressources que vous avez utilisées 
- Enregistrer - mettez un signet à la ressource dont vous avez besoin pour y accéder facilement et rapidement et enregistrez votre stratégie de recherche pour référence future

\section{Services éducatifs}

En plus de publier des ressources organisées, le bulletin mensuel du CANVax offre des mises à jour sur les nouvelles ressources et relève les articles sur les sujets émergents en matière de vaccination. Les balados et les webinaires du CANVax mettent également l'accent sur les sujets émergents, avec des experts de l'ensemble du Canada qui explorent les questions et les initiatives émergentes en matière de vaccination.

\section{Brèves du CANVax}

Le CANVax élabore de nouvelles ressources qui offrent de brefs résumés et mettent en évidence les nouvelles recherches importantes. Les "Brèves du CANVax " sont de brefs articles fondés sur des données probantes visant à informer, à mobiliser et à inspirer les lecteurs, en attirant leur attention sur les questions courantes et émergentes en matière de vaccination, et en signalant les initiatives et les activités dans l'ensemble du Canada qui visent à améliorer l'acceptation et l'adoption de la vaccination.

Dans le présent numéro, nous entamons une série de Brèves du CANVax en mettant d'abord l'accent sur la façon dont I'hésitation à la vaccination est en hausse depuis la disponibilité du Web 2.0 et des médias sociaux, sur le fait que cette hésitation a été associée à une augmentation d'éclosions de maladies évitables par la vaccination, et sur l'identification des meilleures pratiques pour y répondre (17). D'autres Brèves seront publiées dans les prochains numéros du RMTC en 2020.

Les sujets des Brèves sont identifiés par l'équipe du CANVax. L'équipe est formée du personnel de l'ACSP et d'experts en vaccination, ainsi que des docteures Noni MacDonald et Eve Dubé. Les experts sur le sujet choisi sont alors invités à contribuer à la préparation de la Brève. De brèves études de portée des données probantes sont menées et examinées par le Comité d'experts du CANVax, formé d'experts externes en vaccination (18).

\section{Conclusion}

D'énormes progrès fondés sur des études scientifiques ont été réalisés pour répondre à la baisse de la protection collective contre les maladies évitables par la vaccination qui a été constatée partout dans le monde. Le CANVax a été créé pour aider les praticiens de première ligne à se tenir au courant de ces avancées afin d'être en mesure de promouvoir et de maintenir des taux de vaccination plus élevés au Canada. L'un des points forts du site Web du CANVax est qu'il est interactif. Le CANVax invite les commentaires sur son site Web et sur ses produits, et est ouvert aux recommandations sur les sujets spécifiques qui seront abordés dans les futurs webinaires et Brèves du CANVax. Pour obtenir plus de détails, consultez le site Web du CANVax.

\section{Déclaration des auteures}

N. M. - Rédaction de la première ébauche

E. D. - Rédaction - Examen et révision

\section{Conflit d'intérêts}

La Dre MacDonald déclare des subventions provenant de l'Agence de la santé publique du Canada, des Instituts de recherche en santé du Canada, de la Régie de la santé de la Nouvelle-Écosse, du IWK Health Authority et du Réseau canadien de recherche sur l'immunisation. La D're Dubé déclare des subventions provenant de l'Agence de la santé publique du Canada, du ministère de la Santé et des Services sociaux du Québec, du Fonds de la recherche en santé du Québec, des Instituts de recherche en santé du Canada, du Réseau canadien de recherche sur l'immunisation et du Conseil de recherches en sciences humaines du Canada. Les deux auteures sont membres de l'équipe du CANVax.

\section{Remerciements}

Le financement du Centre canadien de ressources et d'échange sur les données probantes en vaccination (CANVax) provient de l'Agence de la santé publique du Canada. Nombreux sont les auteurs, les comités, les partenaires de vaccination et les examinateurs qui contribuent au CANVax, et notamment le secrétariat du CANVax à l'Association canadienne de santé publique (ACSP).

\section{Références}

1. Bloom BR, Lambert PH, editors. The vaccine book, 2 nd ed. Cambridge (MA): Academic Press; 2016.

2. Agence de la santé publique du Canada. Guide canadien d'immunisation. Ottawa (ON): Gouvernement du Canada; 2018 (Accédé 2019-11-03). https://www.canada.ca/fr/santepublique/services/guide-canadien-immunisation.html

3. Programmes de vaccination systématique (et de rattrapage) des provinces et des territoires pour les nourrissons et les enfants au Canada. Ottawa (ON) : Gouvernement du Canada; (Accédé 2019-11-03). https://www.canada.ca/ $\mathrm{fr} /$ sante-publique/services/renseignements-immunisatio n-provinces-et-territoires/programmes-vaccinatio $\mathrm{n}$-systematique-provinces-territoires-nourrissons-enfants.html 
4. Dubé E, Laberge C, Guay M, Bramadat P, Roy R, Bettinger J. Vaccine hesitancy: an overview. Hum Vaccin Immunother 2013 Aug;9(8):1763-73. DOl PubMed

5. Dubé E, Gagnon D, Ouakki M, Bettinger JA, Witteman HO, MacDonald S, Fisher W, Saini V, Greyson D; Canadian Immunization Research Network. Measuring vaccine acceptance among Canadian parents: A survey of the Canadian Immunization Research Network. Vaccine 2018 Jan;36(4):545-52. DOI PubMed

6. Lane S, MacDonald NE, Marti M, Dumolard L. Vaccine hesitancy around the globe: analysis of three years of WHO/ UNICEF Joint Reporting Form data-2015-2017. Vaccine 2018 Jun;36(26):3861-7. DOI PubMed

7. Wielders CC, van Binnendijk RS, Snijders BE, Tipples GA, Cremer J, Fanoy E, Dolman S, Ruijs W, Boot HJ, de Melker $\mathrm{HE}$, Hahné SJ. Mumps epidemic in orthodox religious low-vaccination communities in the Netherlands and Canada, 2007 to 2009. Euro Surveill 2011 Oct;16(41):19989. PubMed

8. Dubey V, Ozaldin O, Shulman L, Stuart R, Maclachlan J, Bromley L, Summers A. Étude et gestion d'une importante éclosion communautaire d'oreillons parmi les jeunes adultes de Toronto, Canada, de janvier 2017 à février 2018. Relevé des maladies transmissibles au Canada 2018 Dec;44(12):351-9. DOI

9. Kershaw T, Suttorp V, Simmonds K, St Jean T. Éclosions de rougeole au sein d'une population ne pratiquant pas la vaccination, Alberta 2013. Relevé des maladies transmissibles au Canada 2014 Jun;40(12):270-8. DOI

10. WHO-UNICEF. WHO vaccine-preventable diseases: monitoring system 2019 global summary. Geneva (CH): World Health Organization; 2019 (Accédé 2019-1103). https://apps.who.int/imDmunization_monitoring/ globalsummary/timeseries/tswucoveragedtp3.html
11. Dubé $E$, MacDonald NE. Addressing vaccine hesitancy and refusal in Canada. CMAJ 2016 Jan;188(1):E17-8. DOI PubMed

12. MacDonald NE; SAGE Working Group on Vaccine Hesitancy. Vaccine hesitancy: Definition, scope and determinants. Vaccine 2015 Aug;33(34):4161-4. DOl PubMed

13. Résistance aux antimicrobiens. Genève $(\mathrm{CH})$ : Organisation mondiale de la Santé; 2018 (Accédé 2019-11-03). https:// www.who.int/news-room/fact-sheets/detail/antimicrobialresistance

14. Dix ennemis que I'OMS devra affronter cette année. Genève (CH): Organisation mondiale de la Santé; 2019 (Accédé 2019-11-03). https://www.who.int/fr/emergencies/tenthreats-to-global-health-in-2019

15. Centre canadien de ressources et d'échange sur les données probantes en vaccination. Ottawa (ON): CANVax; (Accédé 2019-11-03). https://www.canvax.ca/fr

16. About Vaccine Safety Net. Geneva $(\mathrm{CH})$ : World Health Organization; (Accédé 2019-11-03). https://www. vaccinesafetynet.org/vsn/vaccine-safety-net

17. MacDonald NE, Dubé $E$. Promouvoir la résilience vaccinale à l'ère de l'information numérique. Relevé des maladies transmissibles au Canada 2020;46(1):22-7. DOI

18. Comité d'experts du CANVax. Ottawa (ON): CANVax; (Accédé 2019-11-03). https://canvax.ca/fr/comite-dexpertsdu-canvax 